\title{
APPLICATION OF NON-LINEAR $\kappa-\varepsilon$ TURBULENCE MODEL IN FLOW SIMULATION OVER UNDERWATER AXISYMMETRIC HULL AT HIGHER ANGLE OF ATTACK
}

\section{R Sakthivel ${ }^{1}$, $S$ Vengadesan ${ }^{2}$ and $S$ K Bhattacharyya ${ }^{3}$}

${ }^{1}$ Research Scholar, Dept. of Applied Mechanics, Fluid Mechanics Laboratory, IIT Madras, Chennai, India Email: sakthi.smr@gmail.com

${ }^{2}$ Associate Professor, Dept. of Applied Mechanics, Fluid Mechanics Laboratory, IIT Madras, Chennai, India Email: vengades@iitm.ac.in

${ }^{3}$ Professor, Dept. of Ocean Engineering, IIT Madras, Chennai, India, Email: skbh@iitm.ac.in

\begin{abstract}
:
This paper addresses the Computational Fluid Dynamics Approach (CFD) to simulate the flow over underwater axisymmetric bodies at higher angle of attacks. Three Dimensional (3D) flow simulation is carried out over MAYA Autonomous Underwater Vehicle (AUV) at a Reynolds number (Re) of $2.09 \times 10^{6}$. These $3 D$ flows are complex due to cross flow interaction with hull which produces non-linearity in the flow. Cross flow interaction between pressure side and suction side is studied in the presence of angle of attack. For the present study standard $k$ - $\varepsilon$ model, non-linear $k-\varepsilon$ model of turbulence are used for solving the Reynolds Averaged Navier-Stokes Equation (RANS). The non-linear $k-\varepsilon$ turbulence model is validated against DARPA Suboff axisymmetric hull and its applicability for flow simulation over underwater axisymmetric hull is examined. The non-linear $k-\varepsilon$ model performs well in $3 D$ complex turbulent flows with flow separation and reattachment. The effect of angle of attack over flow structure, force coefficients and wall related flow variables are discussed in detail.
\end{abstract}

Keywords: Computational Fluid Dynamics (CFD), Autonomous Underwater Vehicle (AUV), Reynolds averaged Navier-Stokes Equation (RANS), non-linear $k-\varepsilon$ turbulence model

\section{NOMENCLATURE}

$C_{p} \quad$ Time averaged static pressure coefficient;

$U_{I} \quad$ Axial velocity;

$k \quad$ Turbulent kinetic energy;

$F_{n} \quad$ Normal force;

$\mu_{t} \quad$ Turbulent viscosity;

$C_{d} \quad$ Mean drag co-efficient;

$R_{0} \quad$ Radius of the hull section;
$U \quad$ Velocity vector
$\rho \quad$ Density of water
$\varepsilon \quad$ Dissipation rate
$A_{f} \quad$ Frontal area of the hull
$C_{n} \quad$ Mean normal force coefficient
$R \quad$ Radius of point above the hull surface
$R_{\max }$ Maximum radius of the hull

\section{Introduction}

Flow over axisymmetric bodies have wide applications in naval engineering such as torpedoes, AUVs and underwater vehicles etc., Since these vehicles have complicated trajectories during their motion, the flow structure over these bodies is complex in nature. Hence the understanding of such complex flows is necessary to devise methods to enhance the prediction of force coefficient and the vehicle performance. Experimental methods and empirical formulae are widely used to evaluate the hull force coefficients. Though these methods provide a good estimation of force coefficients, it demands high cost at the initial phase of the design process. Flow visualization and cross flow interaction with hull are difficult to study experimentally in the absence of sophisticated instrumentation. Study of flow around a hull at an angle of attack is necessary to evaluate the 
performance of underwater vehicle in various maneuverability conditions. At high angle of attack, the cross flow component of velocity causes the flow separation which produces the non-linear behavior of the flow. Availability of extensive computational resources encourages 3D flow simulation over axisymmetric body which in turn reduces initial experimental cost. Most of the practical flow applications over underwater hulls are transitional and turbulent flows, which are quite complex at higher angle of attacks. Numerical simulation of flow over underwater hull demands optimum grid generation and accurate turbulence modeling. Cross flow interaction over prolate spheroid was investigated experimentally by Wetzel et al (1998). CFD simulation was carried over 6:1 prolate spheroid by Constantinescu et al (2002). Both the studies provide the effect of cross flow component over wall related quantities such as pressure coefficient and wall shear stress. Successful attempts have been reported in the literature for numerical simulations over axisymmetric underwater bodies. Those authors extensively reported the choice of turbulence models for flow simulation over underwater axisymmetric hull forms. Sarkar et al (1997) assessed the performance of $k-\varepsilon$ family of turbulence models by 2D numerical study over different underwater bodies (AFTERBODY1, AFTERBODY2, MS, F-57) at zero angle of attack. Jagadeesh and Murali (2005) studied the application of low Re $k-\varepsilon$ turbulence models. The above mentioned literature gives a detailed study of choice of turbulence models. They also reported that the wall related quantities are in good agreement with experimental results. Alin et al (2010) studied the applicability of LES (Large Eddy Simulation) and DES (Detached Eddy Simulation) for the flow over submarines. They assessed the performance of LES and DES for DARPA Suboff configuration AFF1 (bare hull) and AFF8 (fully appended body).

Most of the above reported studies are for flow over hull at zero angle of attack. However, study of flow characteristics over hull at an angle of attack is important in the design phase of vehicle particularly in propeller design. It determines the vehicle operating range and its maneuverability during its mission. Importantly, the cross flow separation affects the performance of control surface for off design conditions. Experiments are conducted in towing tank to determine the force coefficients for the hull at an angle of attack. These experiments are mainly focused to determine only force coefficients. By suitable CFD methodology it is easier to estimate the flow variables over the hull in addition to force coefficients. Numerical simulation of underwater hull surface at an angle of attack is complicated due its flow structure, thus making the prediction of its flow behavior a challenging task. Phillips et al (2007) and Tyagi and Sen (2006) reported the computational approach to predict the lateral hydrodynamic coefficients at various sway velocities. In their studies different numerical strategies such as numerical drift test, rotating arm test were applied to determine the coefficients of underwater hull. De Barros et al (2008) also developed CFD technique to compute the force coefficients of the bare hull for different angles of attack. The results obtained from the CFD approach were compared with the semi-empirical and experimental values. Jagadeesh et al (2009) made a comparative evaluation between experiments and numerical studies. The lift and drag coefficients were obtained for different angle of attacks in the range of $0^{\circ}$ to $15^{0}$

From the reported literature on the numerical studies for flow over underwater axisymmetric hull surface, it is observed that the three dimensional (3D) flow simulations at an angle of attack are scarce. The 3D simulations are challenging in many aspects such as grid generation, computation and choice of turbulence model. In the present study, 3D flow simulation is carried out for DARPA Suboff to evaluate the performance of non-linear $k$ $\varepsilon$ turbulence model. Flow simulation for MAYA AUV is carried out at high angle of attacks (up to $20^{\circ}$ ). Cross flow interaction over hull is studied extensively. Non-linear behavior of flow variables and hydrodynamic coefficients which arise due to an angle of attack is presented in the following sections.

\section{Mathematical Formulation}

Three dimensional unsteady Reynolds Averaged Navier- Stokes equation for incompressible flow along with continuity equation are given below

$$
\begin{aligned}
& \frac{\partial U_{i}}{\partial x_{i}}=0 \\
& \frac{\partial U_{i}}{\partial t}+\frac{\partial U_{j} U_{i}}{\partial x_{j}}=-\frac{1}{\rho} \frac{\partial p}{\partial x_{i}}+\frac{\partial\left(-\overline{u_{i}^{\prime} u_{j}^{\prime}}\right)}{\partial x_{j}}+v \frac{\partial^{2} U_{i}}{\partial x_{j}^{2}}
\end{aligned}
$$

Application of non-linear $\kappa-\varepsilon$ turbulence model in flow simulation over underwater axisymmetric hull... 
$U_{i}$ is mean velocity in $\mathrm{i}^{\text {th }}$ direction; $\rho$ is density and $\overline{u_{i}^{\prime} u_{j}^{\prime}}$ is Reynolds stress. Two equation $k$ - $\varepsilon$ turbulence model is used for modeling the Reynolds stress. The transport equations for Turbulent kinetic energy $(k)$ and Dissipation rate $(\varepsilon)$ are given by

$$
\begin{aligned}
& \frac{D k}{D t}=-\overline{u_{i}^{\prime} u_{j}^{\prime}} \frac{\partial U_{i}}{\partial x_{j}}-\varepsilon+\frac{\partial}{\partial x_{j}}\left\{\left(\frac{v_{t}}{\sigma_{k}}+v\right) \frac{\partial k}{\partial x_{j}}\right\} \\
& \frac{D \varepsilon}{D t}=-C_{\varepsilon 1} \frac{\varepsilon}{k} \overline{u_{i}^{\prime} u_{j}^{\prime}} \frac{\partial U_{i}}{\partial x_{j}}-C_{\varepsilon 2} \frac{\varepsilon^{2}}{k}+\frac{\partial}{\partial x_{j}}\left\{\left(\frac{v_{t}}{\sigma_{\varepsilon}}+v\right) \frac{\partial \varepsilon}{\partial x_{j}}\right\}
\end{aligned}
$$

where $C_{\varepsilon 1}$ and $C_{\varepsilon 2}$ are constants, $\sigma_{\varepsilon}$ and $\sigma_{k}$ are the turbulent Prandtl numbers for $\varepsilon$ and $k$ respectively. In the present study standard and non-linear $k-\varepsilon$ turbulence models are used. In standard $k-\varepsilon$ model, the Reynolds stress is related to strain rate in a linear fashion as follows:

$$
-\overline{u_{i}^{\prime} u_{j}^{\prime}}=v_{t} S_{i j}-\frac{2}{3} k \delta_{i j}
$$

It does not include anisotropy of turbulence which is the major deficiency of the above model. The deficiency in the model is addressed by introducing the non-linear terms in the Reynolds stress constitutive relation which in general is given as,

$$
-\overline{u_{i}^{\prime} u_{j}^{\prime}}=v_{t} S_{i j}-\frac{2}{3} k \delta_{i j}+\left(\left(-\frac{k}{\varepsilon} v_{t}\right) \times \text { Non }- \text { linear term } s\right)
$$

Non-linear terms in the above mentioned equation are reported in Kimura and Hosoda (2003). The model is mainly used for the complex turbulent flows and for flow over bluff bodies. The model performance for the wall mounted cube is analyzed by Kimura and Hosoda (2003) which is complex three dimensional turbulent flow with flow separation, vortex shedding and flow reattachment. This model satisfies the realizability condition for Reynolds stress. The model coefficients are tuned to satisfy the realizability inequalities for the 3D turbulent flow field. The above mentioned model is incorporated in FLUENT through User Defined Functions (UDFs). Earlier, UDF implementation had been validated for flow past a square cylinder [Ramesh et al (2006)] and for a circular cylinder [Ayyappan and Vengadesan (2008)].

\subsection{Validation}

In addition to the above mentioned validations, in the present study the model is validated for axisymmetric hull, whose stern boundary layer velocity profile and wall related variables are readily available. For the present study, DARPA Suboff experiments by Huang et al (1992), Liu and Huang (1998) are used to validate the nonlinear $k-\varepsilon$ model. The AFF1 configuration is studied by using the present turbulence model and results are compared with the experimental results and LES, DES results by Alin et al (2010). AFF1 is a bare hull configuration. The total length of the hull is $4.36 \mathrm{~m}$ and it has maximum diameter of $0.51 \mathrm{~m}$. Grid and solver parameters are taken from the Alin et al (2010). The free stream air velocity is $41.3 \mathrm{~m} / \mathrm{s}$. The Re based on the length of the vehicle is $12 \times 10^{6}$. For the present study, the typical wall distance of the first grid is taken as 35 $<\mathrm{y}^{+}<60$. From the simulation, pressure coefficient, skin friction coefficient and stern boundary layer velocity profiles are estimated and it is compared with those of the experimental values. Pressure coefficient comparison is shown in Fig.1a. The time averaged static pressure coefficient $C_{p}=p / 0.5 v^{2}$ is compared with those of experimental and LES results. It shows a good agreement with the literature values. Time averaged skin friction coefficient is shown in Fig.1b.

The trend of the curve matches well with that of experimental and LES results. At the stern of the hull, the difference between present prediction and experimental results is small. There is a notable difference in the nose part of the hull by both the LES and present results. The stern boundary layer velocity profile is plotted at $\mathrm{x} / \mathrm{L}=0.877,0.904,0.927,0.956$, and 0.978 as shown in Fig. 2 . The velocity profiles match well with those by experimental values.

Application of non-linear $\kappa-\varepsilon$ turbulence model in flow simulation over underwater axisymmetric hull... 


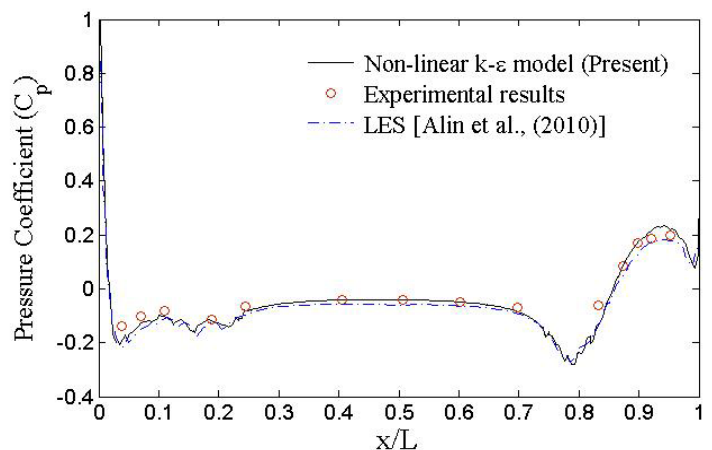

(a)

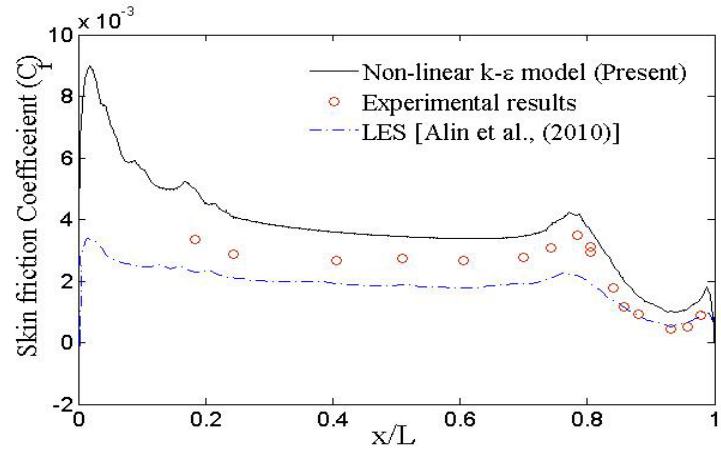

(b)

Figure 1: Comparison of wall variables (a) Time averaged static pressure coefficient (b) Time averaged skin friction coefficient

Table 1: Geometric parameters of MAYA AUV

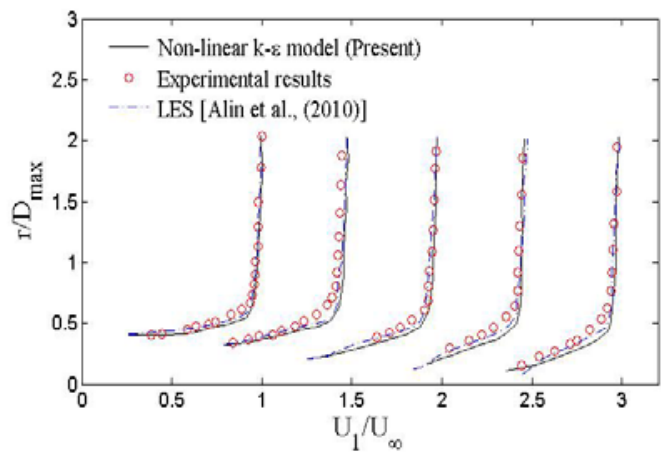

Figure 2: Stern boundary layer velocity profiles

\begin{tabular}{|c|c|}
\hline Description & Values in m \\
\hline Body cylinder length (cl) & 1.246 \\
\hline Nose length (nl) & 0.217 \\
\hline Body cylinder length (cl) & 1.246 \\
\hline Body length (L) & 1.7425 \\
\hline
\end{tabular}

\subsection{Geometry Selection}

For the present study, MAYA AUV geometry is chosen, which is same as reported in De Barros et al (2008). It has a nose, a truncated tail and the geometry as shown in Fig.3. The shape of the body is derived from the Myring parameter [Myring (1976)]. It has a semi-elliptical radius distribution in nose and a cubic radius distribution in tail. The corresponding dimensions of hull are given in Table 1.

\subsection{Domain Description}

O-grid method of grid generation is well suitable for axisymmetric bodies. Body fitted structured O- grid is adopted over the bare hull of MAYA AUV. A typical 3D computational mesh is shown in Fig.4. Domain independence study was carried out separately. Initially the domain size as reported in Sarkar et al (1997) was chosen. Further, two different domains were chosen and results obtained are given in Table 2 . These two domain dimensions have a factor of 1.25 and 0.8 times the initial domain dimension. From the detailed study, it is observed that drag coefficient is almost constant for $100 \%$ and $125 \%$ of the initial domain size. Hence for all further simulations, domain with smaller size was chosen from the point of view of computational cost. The chosen domain has the dimensions as $0.7 \mathrm{~L}$ and $4.5 \mathrm{~L}$ in upstream and downstream respectively and $0.6 \mathrm{~L}$ in radial direction. The typical domain in 2D XY plane is given in Fig.5. Grid independence study was carried out for the chosen domain size. The above computational domain is referred as base domain. 


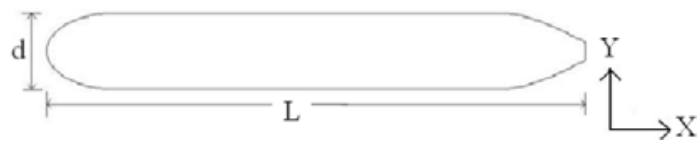

Figure 3: MAYA AUV in XY plane

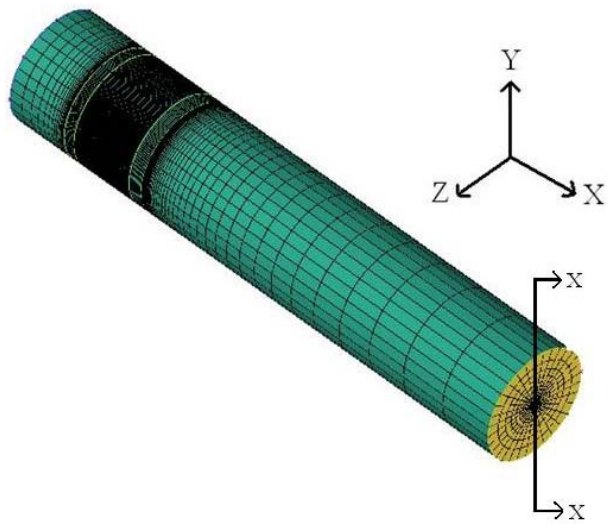

Figure 4: 3D Computational mesh over MAYA

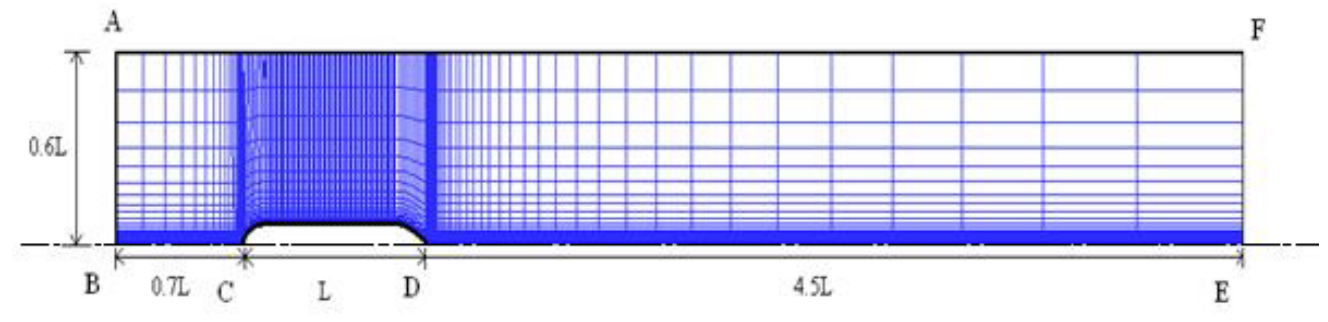

Figure 5: Upper half of computational domain (dimensions at X-X plane)

For angle of attack studies, a larger computational domain (referred as outer domain) is created inside which base computational domain is placed. Dimensions of outer computational domain are given in Fig.6. Base computational domain is drifted in the outer computational domain to obtain required angle of attack. Boundary conditions are imposed on the outer domain corresponding to base domain. The interested variables are monitored in the base computational domain.

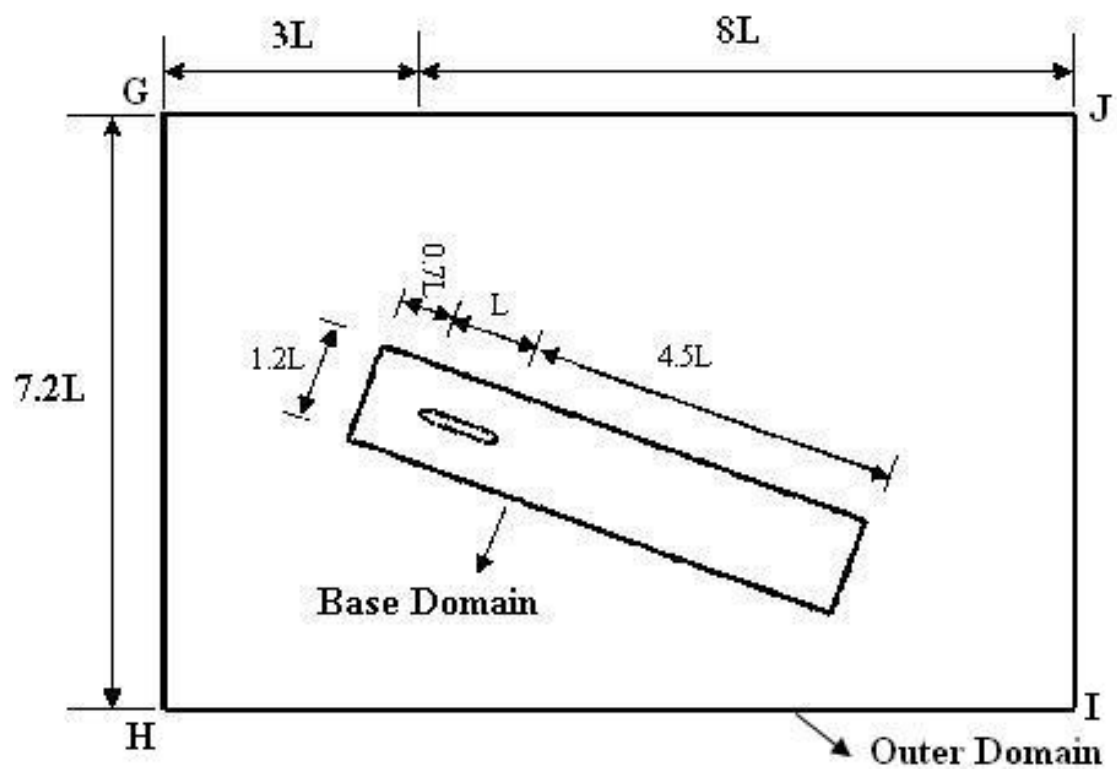

Figure 6: Computational domain for angle of attack studies 
Table 2: Values of drag coefficient for different computational domain sizes

\begin{tabular}{|c|c|c|}
\hline $\begin{array}{c}\text { Domain dimensions (upstream direction } \times \text { downstream } \\
\text { direction } \times \text { radial direction) }\end{array}$ & Drag force $(\mathrm{N})$ & Drag coefficient \\
\hline $0.9 \mathrm{~L} \times 5.6 \mathrm{~L} \times 0.75 \mathrm{~L}$ & 4.2718 & 0.1379 \\
\hline $0.7 \mathrm{~L} \times 4.5 \mathrm{~L} \times 0.6 \mathrm{~L}$ & 4.2411 & 0.1370 \\
\hline $0.6 \mathrm{~L} \times 3.9 \mathrm{~L} \times 0.5 \mathrm{~L}$ & 4.4321 & 0.1431 \\
\hline
\end{tabular}

Four different grids (Grid A, Grid B, Grid C and Grid D) are generated for the grid independence study. Both standard $k-\varepsilon$ model and non-linear $k-\varepsilon$ model are high $R e$ versions. They use the wall functions to compute the flow variables in the near wall region. This requires the $y^{+}$value near the wall to be between 35 to 80 , which is achieved by correctly placing the first grid point away from the wall. Initially, Grid A is chosen as base grid which is relatively coarser. The grid refinement ratio as reported in ITTC manual (1999) is used. The grid refinement ratio $\sqrt{2}$ is applied in each direction to get a fine grid from Grid A. The details of the grids used for the study are given in Table 3. The drag coefficient is observed to be almost same for Grid B, Grid C and Grid D. Hence for all further calculations, Grid D is chosen from computational cost point of view.

Table 3: Values of drag coefficient for different grid sizes

\begin{tabular}{|c|c|c|c|c|c|}
\hline Grid & Refinement Ratio & No .of cells & $\begin{array}{c}\text { Grid Size (streamwise direction } \\
\times \text { radial direction } \times \\
\text { circumferential direction) }\end{array}$ & Drag force (N) & $\begin{array}{c}\text { Drag } \\
\text { coefficient }\end{array}$ \\
\hline Grid A & Base grid & 50176 & $98 \times 16 \times 32$ & 4.80 & 0.155 \\
\hline Grid B & Grid A $\times(\sqrt{ } 2)^{3}$ & 153456 & $139 \times 23 \times 48$ & 4.25 & 0.137 \\
\hline Grid C & Grid $\mathrm{B} \times(\sqrt{ } 2)^{3}$ & 401408 & $196 \times 32 \times 64$ & 4.14 & 0.133 \\
\hline Grid D & Mean of Grid A\&B & 277536 & $168 \times 28 \times 59$ & 4.20 & 0.135 \\
\hline
\end{tabular}

\subsection{Numerical details}

The simulation is carried out for a flow velocity of $1.2 \mathrm{~m} / \mathrm{s}$ (Standard operating speed of MAYA AUV), the Reynolds number $(\mathrm{Re})$ corresponding to the length of the vehicle is $2.09 \times 10^{6}$. The Boundary conditions for the domain are as follows,

- Segment AB (GH for angle of attack studies) is referred as velocity inlet. Along with this the turbulence intensity and the length scales are specified which are same as reported in Sarkar et al (1997)

- No slip condition is imposed on surface of the MAYA which is referred as CD.

- Outflow boundary condition is imposed at the exit EF (JI). At the exit the turbulent dissipation rate $(\varepsilon)$ and turbulent kinetic energy $(k)$ are set to zero.

- The symmetry condition is imposed on the segment AF (GJ\&HI).

Commercial software package, FLUENT ${ }^{\circledR} 6.3$ is used for simulation. SIMPLE algorithm is used for pressure velocity coupling. The computational solution is influenced by the discretization of the governing differential equations. Second order upwind scheme is used for momentum and turbulence quantities. The convergence criterion is set as $10^{-4}$ for continuity, momentum, turbulent kinetic energy and dissipation rate. The time step for unsteady simulation is taken as $10^{-3} \mathrm{~s}$. The numerical details are almost same as reported in Virag et al (2011).

\section{Results and Discussions}

\subsection{Force Coefficients}

Force coefficient is a main parameter which depends on the angle of attack of the hull. Normal force coefficient and drag coefficient are calculated as a function of angle of attack. Normal force coefficient is measured

Application of non-linear $\kappa-\varepsilon$ turbulence model in flow simulation over underwater axisymmetric hull... 
perpendicular to freestream direction. It is obtained by $F_{n}=0.5 C_{n} \rho U_{\infty}{ }^{2} L^{2}$. The normal force coefficient over bare hull is calculated for an angle of attack $(\alpha)$ range of $0^{\circ}$ to $20^{\circ}$ and it is shown in Fig.7.

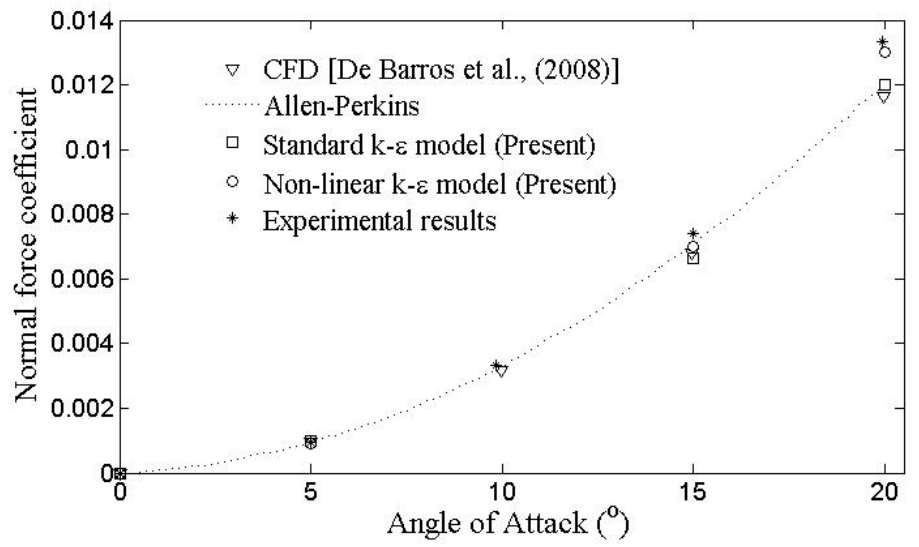

Figure 7: Variation of normal force coefficient with angle of attack $(\alpha)$.

It can be seen that the present simulation has good agreement with the experimental results. At higher angles of attack, the normal force coefficient obtained by the non-linear $k-\varepsilon$ turbulence model shows good agreement than that by the standard $k-\varepsilon$ model. When the angle of attack is increased, the variation of force coefficient became non-linear. There is a significant increase in the slope of force coefficient. This arises due to the cross flow component of velocity, which in turn results in variation of the pressure around the circumference of the hull. The static pressure variation along the hull length is shown in Fig.8.

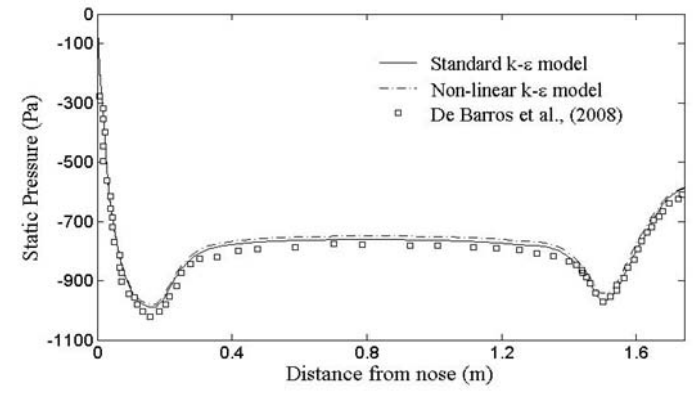

(a) $\alpha=0^{\circ}$

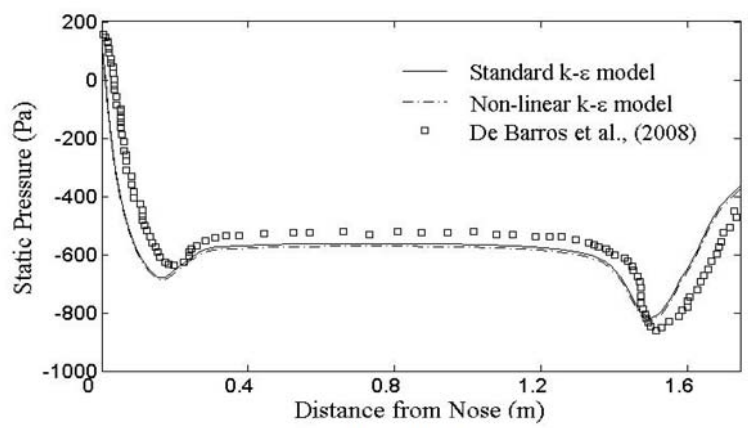

(b) $\alpha=15^{\circ}$

Figure 8: Comparison of pressure variation along the length of the hull at (a) $\alpha=0^{\circ}$ and (b) $\alpha=15^{\circ}$

The comparison between two turbulence models is shown along with the results of De Barros et al (2008). For $\alpha=0^{\circ}$ and $\alpha=15^{\circ}$ case, the static pressure is in good agreement with the literature values. Two pressure valleys are present, which arise due to the shape of the nose and tail section. For $\alpha=15^{\circ}$, there is an offset in predicting the values of the valleys, but the trend of curve is observed to match well with that of the literature. Since non-linear $k-\varepsilon$ model shows good performance in predicting the force coefficients and static pressure, further results obtained by the same model are plotted and discussed. Similarly, the drag coefficient is also computed for various angles of attack. The corresponding values of drag force are tabulated in Table 4. Pressure drag force exhibits variation, whereas the viscous drag force remains constant. There is also an increase in the drag coefficient at higher angle of attack which is solely due to the variation in pressure force.

For $\alpha=20^{\circ}$, pressure drag contributes to drag coefficient by about $193 \%$ of viscous drag. The drag coefficient is obtained by $F_{d}=0.5 C_{d} \rho U_{\infty}{ }^{2} A_{f}$. Here $A_{f}$ refers to the maximum frontal area of the hull. The drag coefficient of hull at zero angle of attack is obtained as 0.13 , which matches with value obtained by empirical formula of Hoerner (1965) and Triantafyllou (2004). 
Table 4: Values of drag coefficient at different angle of attack

\begin{tabular}{|c|c|c|c|c|}
\hline $\begin{array}{c}\text { Angle of } \\
\text { Attack }(\alpha)\end{array}$ & $\begin{array}{c}\text { Pressure } \\
\text { drag }\left(\mathrm{F}_{\mathrm{p}}\right) \mathrm{N}\end{array}$ & $\begin{array}{c}\text { Viscous } \\
\text { drag }\left(\mathrm{F}_{\mathrm{v}}\right) \mathrm{N}\end{array}$ & $\begin{array}{c}\text { Total drag } \\
\left(\mathrm{F}_{\mathrm{d}}\right) \mathrm{N}\end{array}$ & $\begin{array}{c}\text { Drag coefficient } \\
\left(\mathrm{C}_{\mathrm{d}}\right)\end{array}$ \\
\hline $0^{\circ}$ & 0.7785 & 3.4333 & 4.2118 & 0.136 \\
\hline $5^{\circ}$ & 0.9826 & 3.4639 & 4.4465 & 0.144 \\
\hline $15^{\circ}$ & 5.0678 & 3.5819 & 8.6497 & 0.279 \\
\hline $20^{\circ}$ & 7.523 & 3.8811 & 11.407 & 0.368 \\
\hline
\end{tabular}

\subsection{Pressure Variation}

Nomenclature of angular locations $(\theta)$ around the hull in the present study is given in Fig.9. Circumferential pressure variation is plotted at $\mathrm{x} / \mathrm{L}=0.5$ (midsection of the hull) and $\mathrm{x} / \mathrm{L}=0.84$ (starting point of the tail section). These $\mathrm{x} / \mathrm{L}$ stations expected to be important in flow development in the presence of cross flow. As mentioned earlier, the flow becomes non-linear at high angle of attack, which can be easily witnessed by the azimuthal variation of the static pressure as shown in Fig.10. For $\alpha=0^{\circ}$, the pressure variation around hull surface remains constant which clearly implies that the flow is $2 \mathrm{D}$ and symmetric. For $\alpha=5^{\circ}$, the value of static pressure variation around hull is approximately $70 \mathrm{~Pa}$. But this variation becomes more at higher angles. For $\alpha=20^{\circ}$, the magnitude of static pressure variation is around $300 \mathrm{~Pa}$, which contributes for the lift generation. This large circumferential pressure gradient causes the flow around hull surface which is referred as cross flow. Same kind of variation of static pressure around hull was reported in Pantelatos and Mathioulakis (2004). This clearly implies that variation of flow variables is significant in the third dimension. Thus the flow behavior over the hull is highly non-linear and three dimensional at higher angle of attack.

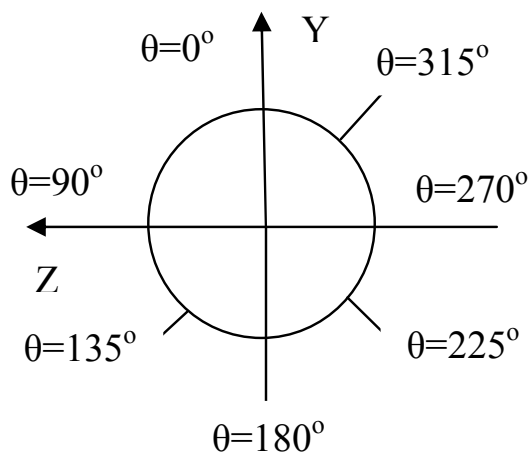

Figure 9: Angular positions around the hull surface

Since the body experiences the cross flow component of velocity due to angle of attack, there is an asymmetry in the flow field around the hull. This asymmetric flow field creates the pressure gradient along the longitudinal and circumferential direction of the hull surface. The pressure variation at various angular locations around the hull is shown in Fig.11.

This shows the magnitude of pressure variation for an angle of attack, $\alpha=20^{\circ}$ is more predominant than that for $\alpha=15^{\circ}$. Even though the pressure difference is present at the nose valley, there is not much change in the pressure at the tail valley except at $\theta=0^{\circ}$ and $\theta=315^{\circ}$. The maximum pressure variation for $\alpha=20^{\circ}$ is of the magnitude of $320 \mathrm{~Pa}$, whereas the variation is $200 \mathrm{~Pa}$ for $\alpha=15^{\circ}$ and zero for $\alpha=0^{\circ}$ case. This variation in the pressure mainly contributes for lift generation at higher angle of attack. Another important feature in the pressure variation at higher angle attack is its contribution to its pressure drag force. Though the viscous drag force is almost constant, there is an increase in the pressure drag force, which contributes to the magnitude of drag coefficient. The magnitude of the pressure difference at the nose for two different angular locations is greater than that at the tail for the same angular locations. This happens due to the movement of stagnation point location along the streamwise direction. For $\alpha=0^{\circ}$, the stagnation point lies exactly at the nose tip of the hull. As the angle of attack increases, the stagnation point moves along the hull length. Since the stagnation point is

Application of non-linear $\kappa-\varepsilon$ turbulence model in flow simulation over underwater axisymmetric hull... 
located only on particular angular location, there is a definite change in velocity magnitude along the circumference of the hull. This attributes to the pressure variation along different angular locations of the nose. As mentioned earlier, the pressure is constant for most part of the mid-body (constant diameter section of the hull) length for any particular angular location. This is clearly observed in all angular locations for $\alpha=0^{\circ}$ and $\alpha=15^{\circ}$. But for an angle of attack of $\alpha=20^{\circ}$, pressure slip is observed, which is defined as pressure variation along the constant radius section of hull caused by circumferential pressure gradients. For $\alpha=20^{\circ}$, pressure slip was observed at two angular locations, $\theta=90^{\circ}$ and $\theta=315^{\circ}$ in the mid-body of the hull.

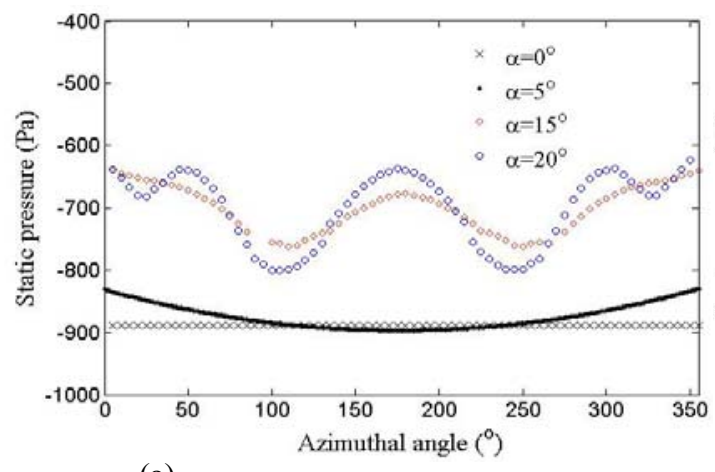

(a)

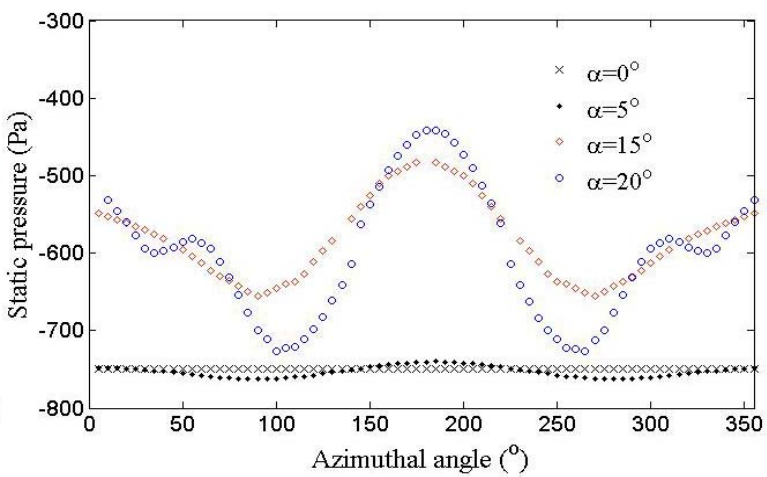

(b)

Figure 10: Variation of static pressure around hull surface for angle of attack $0^{\circ}, 5^{\circ}, 15^{\circ}$ and $20^{\circ}$ at $(\mathrm{a}) \mathrm{x} / \mathrm{L}=0.84$ and (b) $\mathrm{x} / \mathrm{L}=0.5$

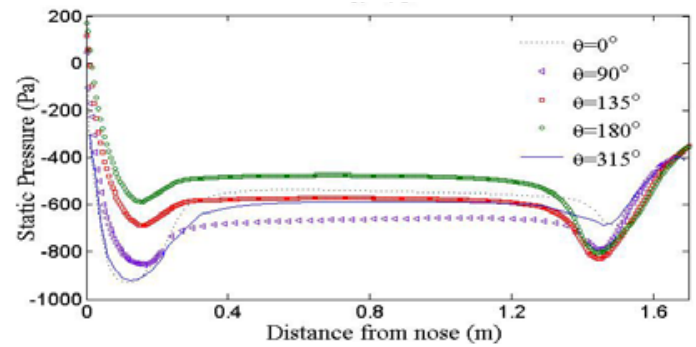

(a)

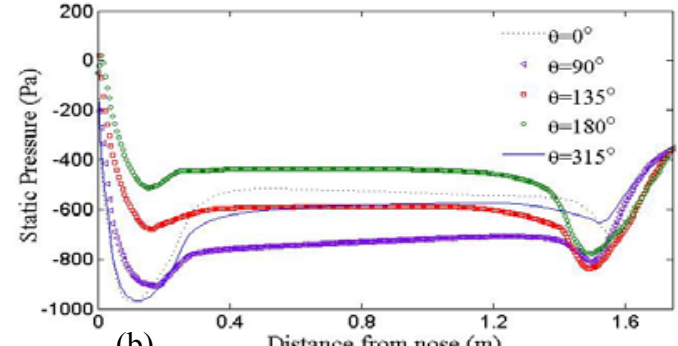

(b)

Figure 11: Pressure variation along longitudinal direction at different angular positions (a) $\alpha=15^{\circ}$ and (b) $\alpha=20^{\circ}$.

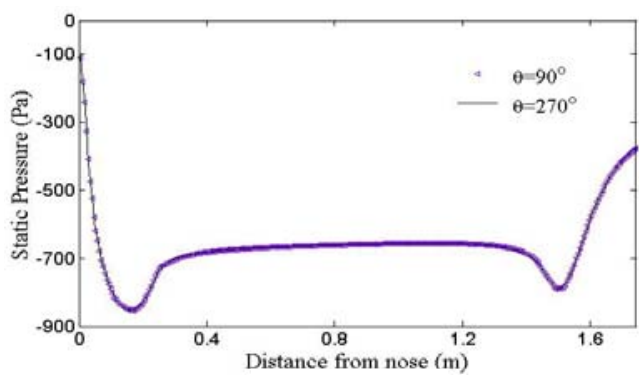

(a) $\alpha=15^{\circ}$

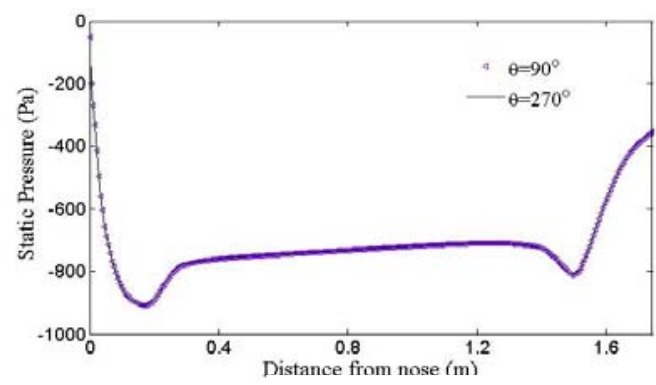

(b) $\alpha=20^{\circ}$

Figure 12: Comparison of pressure at angular position $\theta=90^{\circ}$ and $\theta=270^{\circ}$ for (a) $\alpha=15^{\circ}$ (b) $\alpha=20^{\circ}$

At angular positions $\theta=90^{\circ}$ and $\theta=270^{\circ}$, the pressure variation for higher angle of attack, $\alpha=15^{\circ}$ and $\alpha=20^{\circ}$ is shown in Fig.12. Interestingly, the pressure is same in both the angular locations along the length of the hull. Hence the XZ plane at $\theta=90^{\circ}$ divides the flow field into two major regions namely, Pressure Side (PS) and Suction Side (SS). The study of pressure side and suction side provide the structure of the flow field at higher angle of attack and the source of force coefficients, cross component force. This includes the determination of 
boundary layer velocity profile and study of vortex structure over the hull and pressure variation in streamwise and crosswise direction.

\subsection{Velocity distribution}

The boundary layer profile is plotted between the normalized axial velocity $\left(U_{I} / U_{\infty}\right)$ and the normalized distance $\left[\left(R-R_{0}\right) / R_{\max }\right]$, which is perpendicular to the hull surface. The boundary layer velocity profile at different stations viz. $\mathrm{x} / \mathrm{L}=0.5,0.75,0.87$ (starting of the tail section) and 0.93 (midsection of the tail) along the streamwise direction of the body on both PS and SS for $\alpha=0^{\circ}, \alpha=15^{\circ}$ and $\alpha=20^{\circ}$ are shown in Fig. 13 .

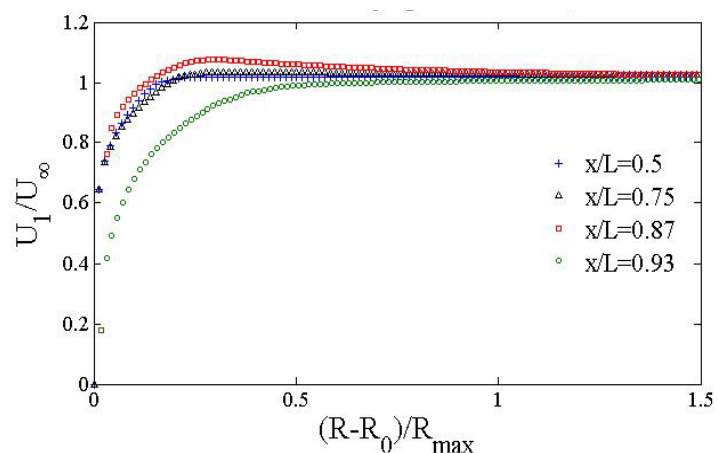

(a) $\alpha=0^{\circ}$

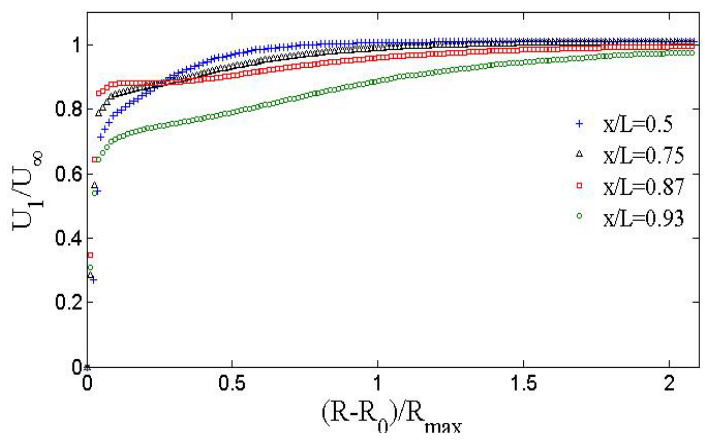

(c) $\alpha=15^{\circ}$ suction side

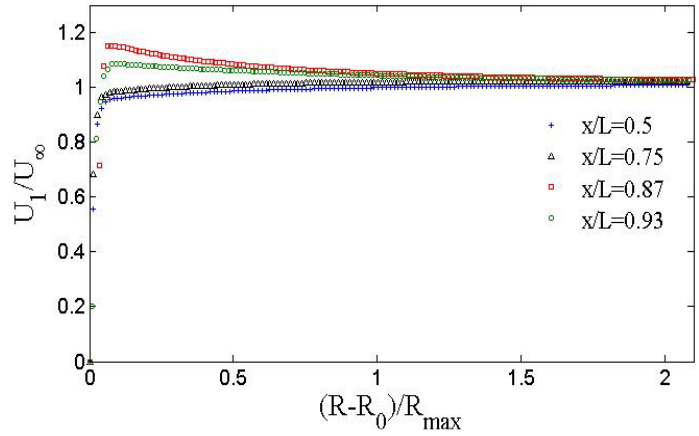

(b) $\alpha=15^{\circ}$ pressure side

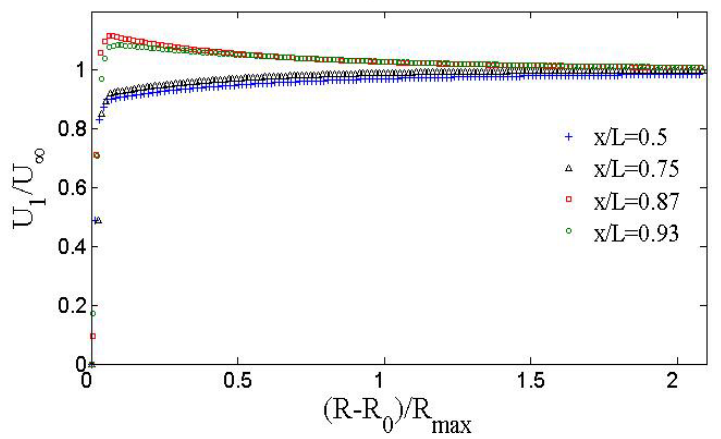

(d) $\alpha=20^{\circ}$ pressure side

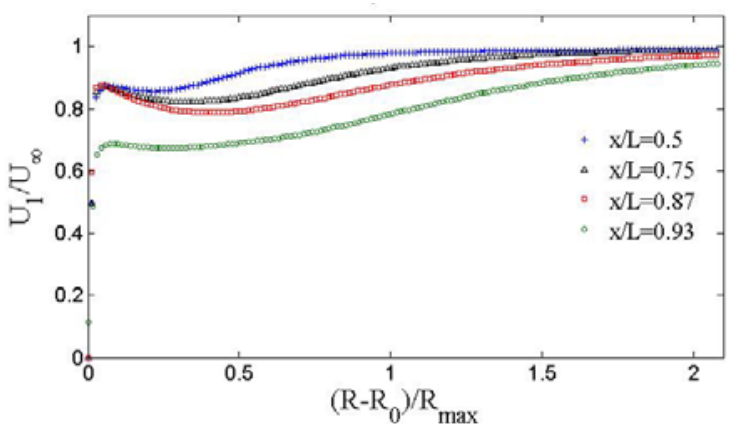

(e) $\alpha=20^{\circ}$ suction side

Figure 13: Comparison of boundary layer velocity profile at different streamwise stations for $\alpha=0^{\circ}, \alpha=15^{\circ}$ and $\alpha=20^{\circ}$.

Velocity profiles at these stations provide flow evolution over the hull. For $\alpha=0^{\circ}$, the normalized axial velocity reaches unity when the normalized distance is 0.5 . Station $\mathrm{x} / \mathrm{L}=0.87$ is located in the beginning of the tail section. The flow accelerates for a short distance and hence the normalized axial velocity exceeds one much earlier. However, at $\mathrm{x} / \mathrm{L}=0.93$, the slope of the velocity gradient is shallow when compared to that of other 
stations. This represents the thickening of boundary layer at the midsection of the tail. For $\alpha=0^{\circ}$ case, since the flow is symmetric, the boundary layer profile remains same on both sides of the hull. A clear change in the velocity profile is observed on the pressure and suction side for higher angle of attack cases. For $\alpha=15^{\circ}$ case, the pressure side boundary layer becomes thin and it has a steep velocity gradient. It is observed that PS boundary layer thickness is same in all stations. Since the flow stagnation point is located on PS, there is a favorable pressure gradient present at $\mathrm{x} / \mathrm{L}=0.87$ and $\mathrm{x} / \mathrm{L}=0.93$, which makes the tail boundary layer thin (Fig. 13b). Conversely, at different $\mathrm{x} / \mathrm{L}$ variation in the velocity profile on SS of the hull is observed. Due to adverse pressure gradient in the streamwise direction on the SS, boundary layer growth begins after particular station along the body. In Fig. $13 \mathrm{c}$, for $\alpha=15^{\circ}$ at $\mathrm{x} / \mathrm{L}=0.5$ and $\mathrm{x} / \mathrm{L}=0.75$, the boundary layer thickness is 0.5 (in terms of the normalized distance). After $\mathrm{x} / \mathrm{L}=0.87$, the boundary layer thickness has increased significantly. More interestingly, the normalized velocity reaches unity for the normalized distance of 2 , which is $400 \%$ greater when compared to the boundary layer thickness for $\alpha=0^{\circ}$. This regime of diffused vorticity moves towards the nose of the hull at the same station for an angle of attack $\alpha=20^{\circ}$. Similar kind of profiles are observed for $\alpha=20^{\circ}$ on PS (Fig. 13d) and SS (Fig. 13e) of hull. There is no noticeable variation in the velocity profile on the pressure side of the hull. It behaves in a similar way as in $\alpha=15^{\circ}$ case.

At $x / L=0.5$, the normalized axial velocity reaches unity when the normalized distance is beyond 0.5 . At $x / L=0.5$, the boundary layer thickness is higher for $\alpha=20^{\circ}$, when compared to the same for lower angle of attacks. For lower angles of attack the boundary layer thickness starts increasing near the tail, and it moves towards nose when angle of attack is increased. Comparison of velocity profiles is represented in Fig.14.

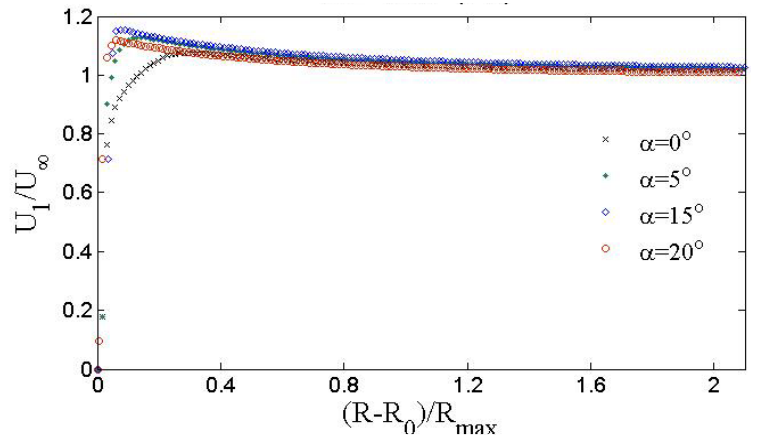

(a) Pressure side at $\mathrm{x} / \mathrm{L}=0.87$

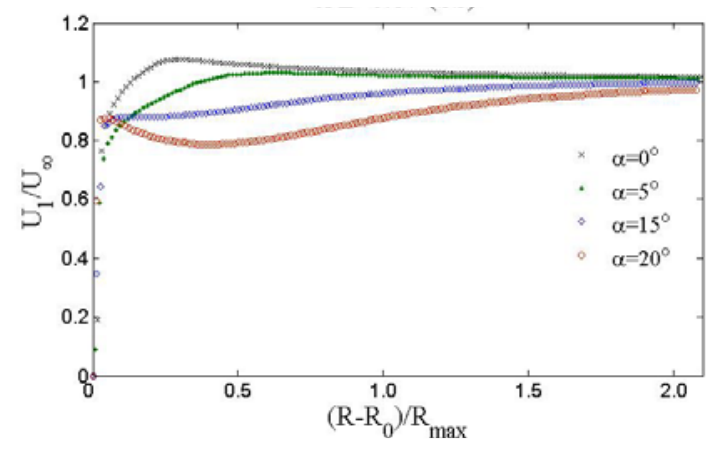

(b) Suction side at $\mathrm{x} / \mathrm{L}=0.87$

Figure 14: Boundary layer velocity profile for different angle of attack (a) Pressure side (b) Suction side

The relative change in the boundary layer velocity profile on PS and SS for all cases of angle of attack are plotted at the station $\mathrm{x} / \mathrm{L}=0.87$. This station represents the starting section of tail, where variations of flow variables are predominant. This clearly shows the evaluation of boundary layer with the increase in angle of attack. On the PS, the variation is not that significant and it remains identical after $\alpha=5^{\circ}$. The velocity profiles on SS are shown in the Fig.14b, which implies the growth of boundary layer as the angle of attack increases. For $\alpha=15^{\circ}$ and $\alpha=20^{\circ}$, the variation is more predominant because of the presence of the negative slope of the velocity gradient in the flow regime. The negative slope is observed at the other station, $\mathrm{x} / \mathrm{L}=0.87$ for $\alpha=20^{\circ}$. The boundary layer extends after the normalized distance of 2 for $\alpha=20^{\circ}$.

\subsection{Streamlines}

Streamlines are used to study the nature of fluid motion in complex flow field. Streamline pattern at different sections of the hull provides details of flow development over the hull length. From the previous discussion, it is obvious that cross flow component of velocity produces non-linearity in flow field. Cross flow arises due to angle of attack of the hull. The typical flow structures around axisymmetric bodies were reported in Wetzel et al (1998) and Pantelatos and Mathioulakis (2004). Experimental studies show that there is a flow separation on Suction Side (Leeward Side). As a consequence of this separation, two counter acting vortices are formed on the suction side of the hull. These vortices are transported further downstream along the hull surface. Pantelatos and Mathioulakis (2004) reported the typical flow pattern over hull at higher angle of attack by oil flow visualization which shows the cross flow interaction and the separation line. In the present study, limiting streamlines are drawn over MAYA hull. Volumetric streamlines provide the structure of 3D flow pattern. These streamlines are

Application of non-linear $\kappa-\varepsilon$ turbulence model in flow simulation over underwater axisymmetric hull... 
used to study the flow nature over the hull. Volumetric limiting streamlines over the MAYA AUV for different angle of attacks are shown in Fig. 15 . For $0^{\circ}$ case the streamlines structure shows that the flow is symmetric around the hull surface. Streamlines are parallel to freestream direction in the absence of cross flow. As stated before, the cross flow interaction with hull is significant for higher angle of attacks. In the presence of cross flow, streamlines are curved around the hull. It is seen for $\alpha=5^{\circ}$ case. Streamlines for $\alpha=15^{\circ}$ and $\alpha=20^{\circ}$ case are shown in Fig.15. (c) \& (d). It is observed that, streamlines are tending to converge on the suction side of the hull which is referred as the separation line. Circumferential pressure gradient causes the streamlines to move towards the suction side. The concentrated streamline region is observed over the suction side of the hull.
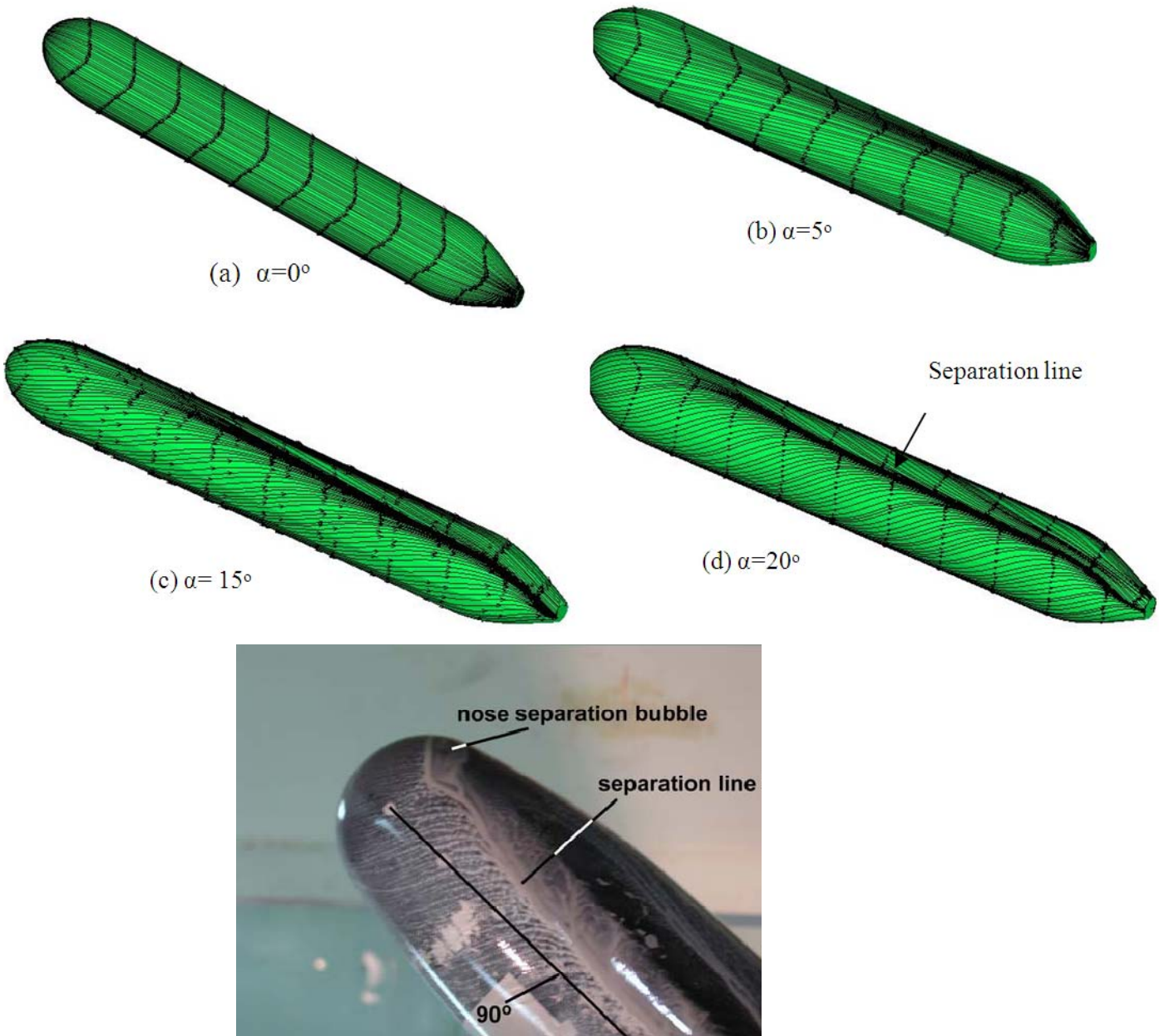

(e) Flow visualization by Pantelatos and Mathioulakis (2004)

Figure 15: Volumetric streamline patterns over MAYA AUV at different angle of attack

This region starts just after the nose section and extends to remaining part of the hull. Converged streamlines appear as a thick black line over the hull which indicates the separated flow region and the movement of vortices along the downstream (Fig. 15d). The separation line is observed for $20^{\circ}$ case where the separation starts just after the nose section and separation is delayed for $15^{\circ}$ case. It is evident from the length of the separation line. The length of the separation line is higher for $20^{\circ}$ case. Flow pattern over axisymmetric body by oil flow visualization reported by Pantelatos and Mathioulakis (2004) is shown in Fig.15e. Present streamline patterns matches well with those of the experimental pattern. Streamlines along the plane YZ for angle of attack $\alpha=15^{\circ}$ is shown in Fig. 16 . 


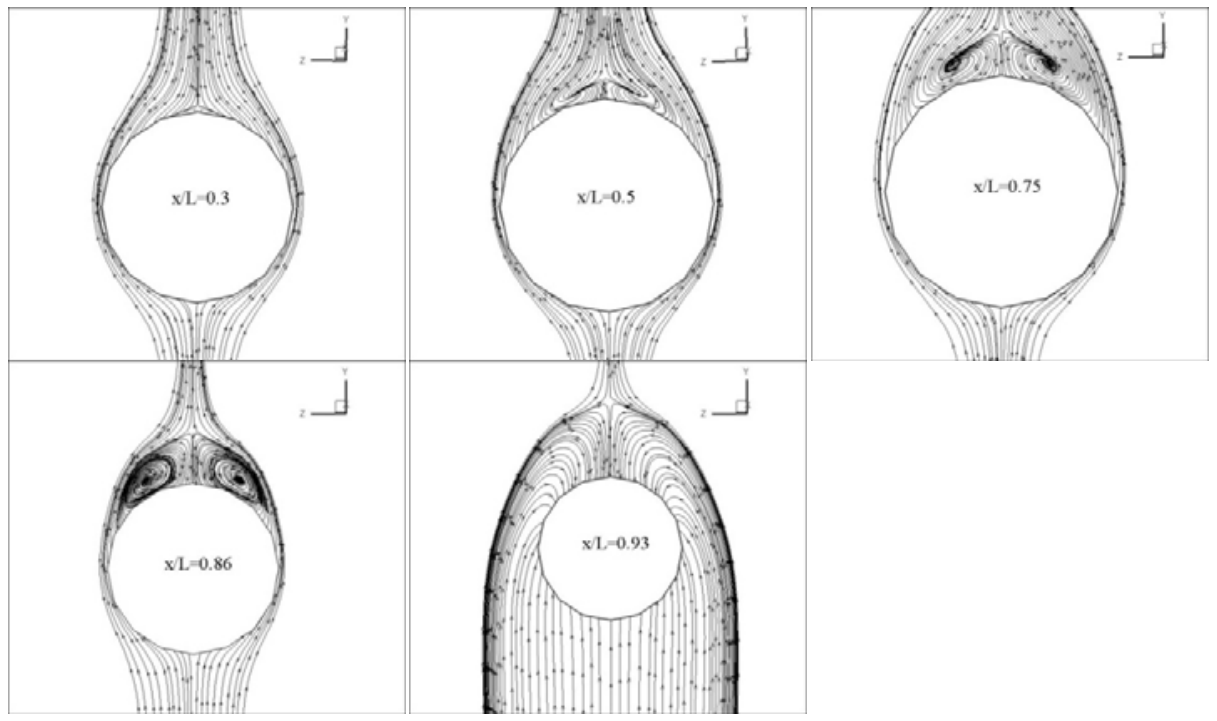

Figure 16: Streamline patterns at different sections for $\alpha=15^{\circ}$

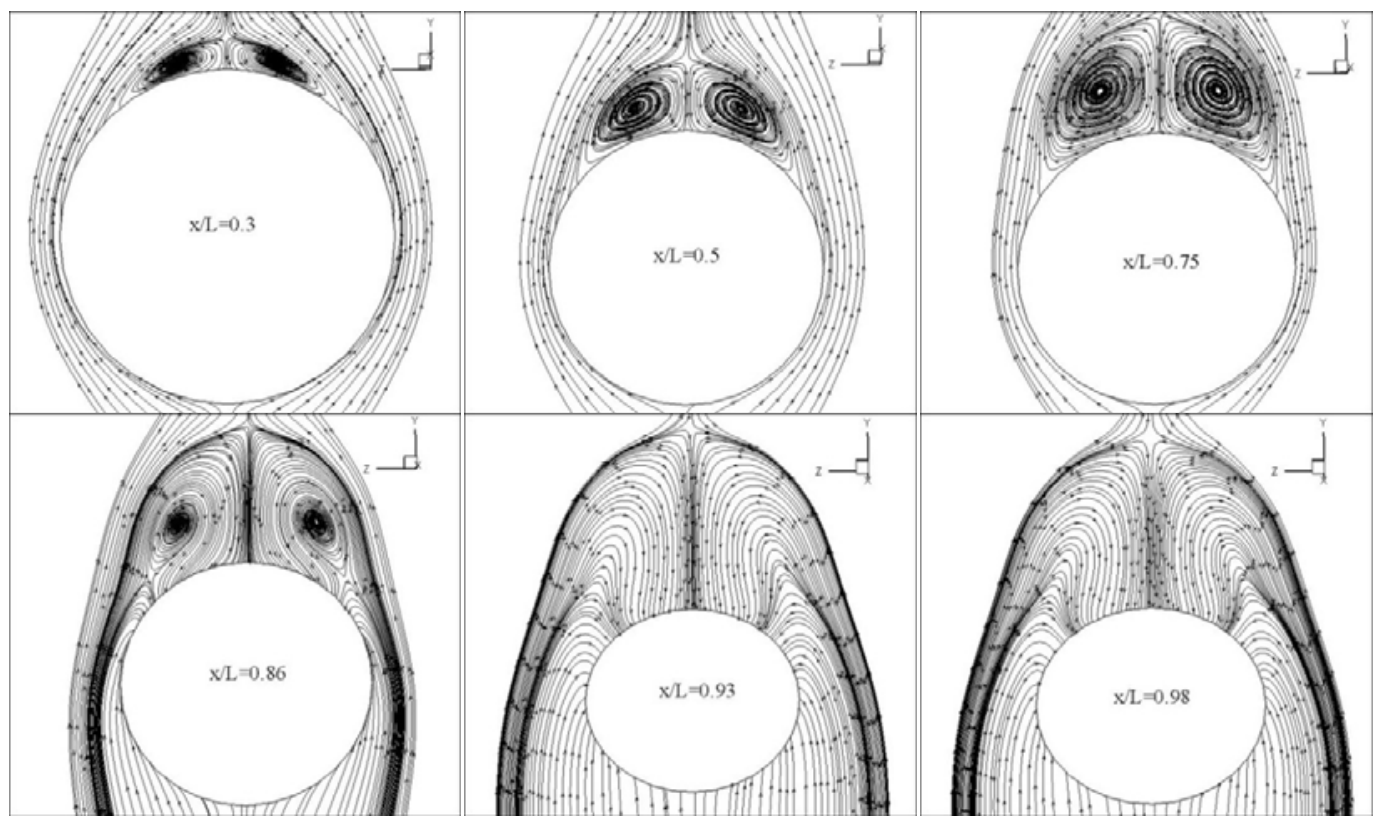

Figure 17: Streamline patterns at different sections for $\alpha=20^{\circ}$

At $\mathrm{x} / \mathrm{L}=0.3$, the flow is fully attached to hull. At $\mathrm{x} / \mathrm{L}=0.5$ and 0.75 , the flow begins to separate from the hull which is spotted by small patches of swirling streamline. Further downstream, the flow is fully separated and it forms the symmetric vortex pair. At $\mathrm{x} / \mathrm{L}=0.86$, the symmetric vortex pairs are spotted. Due to cross flow interaction, the vortex cores expands out at $\mathrm{x} / \mathrm{L}=0.93$. Streamlines for different sections along the length for $\alpha=20^{\circ}$ case are shown in Fig. 17 . At $\mathrm{x} / \mathrm{L}=0.3$, the flow pattern indicates the flow separation from the suction side of the hull. This creates the two counter acting vortex, which gains its strength as it proceeds towards downstream of the hull. This is clearly observed in further $\mathrm{x} / \mathrm{L}$ stations. Correspondingly, the vortex core moves away from the hull surface which creates the circumferential pressure gradient to interact with the vortex core. At $\mathrm{x} / \mathrm{L}=0.86$, the cross interaction with vortex core is observed. When the cross flow interaction with vortex core is predominant, the vortex core diffuses and getting larger. This is observed at $\mathrm{x} / \mathrm{L}=0.93$ and $\mathrm{x} / \mathrm{L}=0.98$. As 
reported earlier, the diffused region is of the order of hull diameter near the tail. Separation happens earlier for $20^{\circ}$ case when compared to that for lower angle of attack cases.

\section{Conclusions}

Intensive computational simulations are carried out for flow over the axisymmetric body for various angles of attack. Initially, non-linear $k-\varepsilon$ turbulence model is validated against DARPA Suboff axisymmetric body. Nonlinear $k-\varepsilon$ model performs well for 3D turbulent flows with flow separation and reattachment. It predicts the force coefficients better when compared to those by standard $k-\varepsilon$ turbulence model particularly at higher angle of attack. Non-linear variation in normal force coefficient at higher angle of attack is observed which is caused by pressure variation between suction side and pressure side. The effect of cross flow component of velocity over drag coefficient is calculated which increases the drag coefficient for higher angle of attack cases. The viscous drag is almost constant for all cases, whereas the pressure drag is $193 \%$ higher than the viscous drag for $\alpha=20^{\circ}$. Since the cross flow component produces the pressure variation on the hull surface, the streamwise and circumferential variation of pressure is calculated, which significantly contributes for the non-linear behavior of the flow. The circumferential pressure gradients play a significant role in flow variables at higher angle of attack. For lower angles of attack, the flow separation happens near the tail of hull, which moves towards nose when the angle of attack is increased. This is observed to be due to higher circumferential pressure gradients. The 3D flow simulation was effectively used to study the flow behavior over underwater hull at higher angle of attack.

\section{Acknowledgement}

Authors express their gratitude for Naval Research Board (NRB), India for their financial and technical support during this work.

\section{References}

Alin, N., Bensow, R.E., Fureby, C., Huuva, T. and Svennberg U. (2010): Current capabilities of DES and LES for submarines at straight course, Journal of Ship Research, vol.54, No. 3, pp. 184-196.

Ayyappan,T. and Vengadesan, S.(2008): Three dimensional unsteady simulation of turbulent flow past circular cylinder, Numerical Heat Transfer, Vol. 54, No. 2, pp.221-234.http://dx.doi.org/10.1080/10407780802084694

Constantinescu, G.S., Pasinato, H., Wang, Q.Y., Forsythe, J.R. and Squires, K.D (2002): Numerical Investigation of Flow past a Prolate Spheroid, Journal of Fluid Engineering, Vol.124, pp.904-910. http://dx.doi.org/10.1115/1.1517571

De Barros, E. A., Pascoal, A. and E de Sa. (2008): Investigation of a method for predicting AUV derivatives, Ocean Engineering, Vol.35, pp.1627-1636.

http://dx.doi.org/10.1016/j.oceaneng.2008.08.008

De Barros, E. A., Dantas, L. D., Pascoal, A. M. and E de Sa. (2008): Investigation of normal force and moment coefficients for an AUV at nonlinear angle of attack and sideslip range, Journal of Oceanic Engineering, Vol. 33, no. 4, pp. 583-579. http://dx.doi.org/10.1109/JOE.2008.2004761

Hoerner, S.F. (1965): Fluid dynamics drag, Hoerner Fluid Dynamics, Brick Town. Huang, T.T., Liu, H.L., Groves. N.C., Forlini, T.J., Blanton, J. and Gowing, S. (1992): Measurements of flows over an axisymmetric body with various appendages (DARPA SUBOFF Experiments), Proceedings 19th symposium on Naval Hydrodynamics, Seoul, Korea.

ITTC quality manual (1999): Uncertainty analysis in CFD and guidelines for RANS codes, Interim Recommended Procedure Prepared by Resistance Committee of 22nd ITTC.

Liu, H. and Huang, T. (1998): Summary of DARPA SUBOFF experimental program, Naval surface warfare center carderock division technical report, CRDKNSWC/HD- 1298-11.

Jagadeesh, P., Murali, K. and Idichandy V. G. (2009): Experimental investigation of hydrodynamic force coefficients over AUV hull form, Ocean Engineering, Vol. 36, pp. 113-118.

http://dx.doi.org/10.1016/j.oceaneng.2008.11.008

Jagadeesh, P. and Murali, K. (2005): Application of low-Re turbulence models for flow simulations past underwater vehicle hull forms, Journal of Naval Architecture and Marine Engineering, Vol.1, 41-54.

Kimura, I. and Hosoda, T. (2003): A nonlinear k- model with realizability for prediction of flows around bluff bodies, International Journal of Numerical Methods in Fluids, Vol. 42, pp. 817-837. http://dx.doi.org/10.1002/fld.540 
Myring, D.F. (1976): A theoretical study of body drag in subcritical axisymmetric Flow, Aeronautical Quarterly, vol. 27, no. 3, pp. 186-194.

Pantelatos, D.K. and Mathioulakis, D.S. (2004): Experimental flow study over a blunt-nosed axisymmetric body at incidence, Journal of Fluids and Structures, Vol.19, pp. 1103-1115. http://dx.doi.org/10.1016/j.jfluidstructs.2004.07.004

Phillips, A., Furlong, M. and Turnock S. R. (2007): The use of computational fluid dynamics to determine the dynamic stability of an autonomous underwater vehicle, in Proceedings of 10th Numerical Towing Tank Symposium, Hamburg, Germany.

Ramesh, V., Vengadesan, S. and Narasimhan, J.L. (2006): 3D Unsteady RANS simulation of turbulent flow over bluff body by non-linear model, International Journal for Numerical Methods in Heat and Fluid Flow, Vol.26, No. 6, pp.660-673. http://dx.doi.org/10.1108/09615530610679048

Sarkar, T., Sayer, P.G. and Fraser S.M. (1997): A study of autonomous underwater hull forms using computational fluid dynamics, International Journal of Numerical Methods in Fluids, Vol.25, pp. 1301-1313. http://dx.doi.org/10.1002/(SICI)1097-0363(19971215)25:11<1301::AID-FLD612>3.0.CO;2-G

Sarkar, T., Sayer, P.G. and Fraser S.M. (1997): Flow simulation past axisymmetric bodies using four different turbulence models, Applied Mathematical Modelling, Vol.21, pp.783-792. http://dx.doi.org/10.1016/S0307904X(97)00102-9

Triantafyllou, S.M. (2004): Maneuvering control of surface and underwater vehicles, Lecture Notes for MIT Ocean Engineering Course, pp.25-26. http://ocw.mit.edu/courses/mechanical-engineering/2-154-maneuveringand-control-of-surface-and-underwater-vehicles-13-49-fall-2004/lecture-notes/

Tyagi, A. and Sen, D. (2006) : Calculation of transverse hydrodynamic coefficients using computational fluid dynamic approach, Ocean Engineering, Vol.33, pp.798-809. http://dx.doi.org/10.1016/j.oceaneng.2005.06.004

Virag, M., Vengadesan, S. and Bhattacharyya, S.K. (2011): Translational added mass of axisymmetric underwater vehicles with forward speed using computational fluid dynamics, Journal of Ship Research, Vol. 55, No. 3, pp. 185-195.

Wetzel, T.G., Simpson, R.L. and Chesnakas, C.J. (1998): Measurement of three-dimensional cross flow separation, American Institute of Aeronautics and Astronautics, Vol. 36, No. 4, pp. 557-564. 